\title{
Gamma-ray Spectral and Morphological study of HESS J1912+101 observed by MAGIC and Fermi-LAT
}

Tsutomu Nagayoshi

Institute for Cosmic Ray Research

E-mail: tsutomu@icrr.u-tokyo.ac.jp

David M. Green*

Max-Planck-Institut für Physik

E-mail: damgreen@mpp.mpg • de

\section{Francesco de Palma}

Istituto Nazionale di Fisica Nucleare, Bari

E-mail: francesco.depalma@ba.infn. it

\section{for the MAGIC and Fermi-LAT Collaborations ${ }^{\dagger}$}

The shock waves of supernova remnants (SNRs) are prime candidates for cosmic-ray accelerators. A general feature of the blast wave is an extended shell visible in $\mathrm{GeV}$ and $\mathrm{TeV}$ gamma rays. HESS J1912+101 is a bright unassociated extended TeV source with a shell-like structure. This source has no counterparts at other wavelengths so far, hence it is still labeled as 'SNR candidate'. We are going to present the results of a deep observation campaign with the MAGIC telescopes together with 10 years of PASS 8 Fermi-LAT data. In order to analyze the data, we use a new 2D likelihood software called SkyPrism that allows parametrization of the morphology measured by MAGIC. We measure a joint Fermi-MAGIC flux spectrum covering three decades of energy. This spectrum is well fitted by a power-law with a hard spectral index and a cut-off at a couple $\mathrm{TeV}$.

36th International Cosmic Ray Conference -ICRC2019-

July 24th - August 1st, 2019

Madison, WI, U.S.A.

\footnotetext{
* Speaker.

†https://magic.mpp.mpg.de/ and https://glast.sites.stanford.edu/. For collaboration list see PoS(ICRC2019)1177
} 


\section{Introduction}

Very High Energy (VHE) $\gamma$ rays, typically from $50 \mathrm{GeV}$ to tens of $\mathrm{TeV}$, are excellent tracers of particle acceleration and critical in determining the nature of unassociated and unidentified $\mathrm{TeV}$ sources through measuring morphological and spectral information. Additionally, high energy (HE) $\gamma$ rays, typically from $100 \mathrm{MeV}$ to hundreds of $\mathrm{GeV}$, are further able to measure morphology and spectral information providing a continuous spectral observation, when combined with VHE data, for over 5 decades in energy. These combined measurements give a unique view into the underlying particle population of the acceleration region and can reveal the nature of unassociated and unidentified $\gamma$-ray sources. This non-thermal radiation is produced by either the underlying high-energy (HE) electron population at the acceleration location through inverse Compton (IC) scattering on local photon fields, non-thermal bremsstrahlung, and synchrotron radiation or the underlying high energy proton population interacting with the local interstellar medium (ISM) and producing $\gamma$ rays via $\pi^{0}$ production.

Table 1: Summary of HESS J1912+101 morphology and spectral results from the H.E.S.S. Collaboration [2].

\begin{tabular}{cc|cc}
\hline \hline \multicolumn{2}{c|}{ Morphology Parameters } & \multicolumn{2}{c}{ Spectral Parameters } \\
\hline Variable & Value & Variable & Value \\
\hline $\mathrm{RA}\left[{ }^{\circ}\right]^{\mathrm{a}}$ & $288.29_{-0.02}^{+0.03}$ & {$\left[\mathrm{E}_{\text {min }}, \mathrm{E}_{\text {max }}\right][\mathrm{TeV}]$} & {$[0.68,61.90]$} \\
$\mathrm{DEC}\left[^{\circ}\right]^{\mathrm{a}}$ & $10.19_{-0.02}^{+0.03}$ & $\mathrm{E}_{0}[\mathrm{TeV}]$ & 2.25 \\
$R_{\text {in }}\left[{ }^{\circ}\right]^{\mathrm{b}}$ & $0.32_{-0.02}^{+0.02}$ & $\mathrm{~N}_{0}\left[\mathrm{~cm}^{-2} \mathrm{~s}^{-1} \mathrm{TeV}^{-1}\right]$ & $\left(4.82 \pm 0.43_{\text {stat }} \pm 1.45_{\text {sys }}\right) \times 10^{-13}$ \\
$R_{\text {out }}\left[{ }^{\circ}\right]^{\mathrm{b}}$ & $0.49_{-0.03}^{+0.04}$ & $\Gamma$ & $2.56 \pm 0.09_{\text {stat }} \pm 0.02_{\text {sys }}$ \\
\hline \hline
\end{tabular}

HESS J1912+101 is an extended unassociated Galactic TeV source, located near the W49B supernova remnant $(\mathrm{SNR})$ at $(\mathrm{RA}, \mathrm{DEC})=\left(288.29^{\circ}, 10.19^{\circ}\right)$. It was discovered in 2008 by the H.E.S.S. Collaboration and initially believed to be a pulsar wind nebula (PWN) associated with an old and energetic pulsar, PSR J1913+1011, located at the center of the extension[1]. Recent results by the H.E.S.S. Collaboration, utilizing an increase of exposure, reveal a shell-like morphology and a spectral energy distribution (SED) well fit with a simple power-law[2]. The morphological and spectral parameters of these recent results are shown in Table 1. These results are more indicative of HESS J1912+101 being a young TeV SNR much like RX J1713.7-3946, RCW 86 or SN 1006 but a PWN associated with PSR J1913+1011 can still not be ruled out. HESS J1912+101 remains unassociated due to the lack of a counterpart in other wavelengths such as radio and X-rays which would greatly help in distinguishing between a SNR or PWN.

We present the results of a joint analysis with The Major Atmospheric Gamma Imaging Cerenkov (MAGIC) telescopes and Large Area Telescope (LAT) on board the Fermi Gamma Ray Space Telescope of HESS J1912+101. Using $\sim 86$ hours of high quality MAGIC data and 128 months of the latest PASS 8 Fermi-LAT data we are able to detect and measure an extended source with compatible morphology to previous studies. 


\section{MAGIC Analysis and Results}

MAGIC is a system of two $17 \mathrm{~m}$ diameter Imaging Atmospheric Telescopes (IACTs) located at on altitude of $2200 \mathrm{~m}$ a.s.l on the Canarian island of La Palma, Spain, at the Roque de los Muchachos Observatory. The MAGIC telescopes operate in a stereoscopic mode, to reduce cosmic-ray background events, and record Cerenkov light produced in Extensive Air Showers from VHE $\gamma$ rays. HESS J1912+101 was observed from May 2016 to August 2017 in the 'Wobble'[3] mode, with a wobble offset of $0.6^{\circ}$ to account for the source extension, for a total of $\sim 130$ hours. This data is further cleaned by removing events coinciding with either bad weather and technical problems using contemporaneous atmospheric monitoring and telescope monitoring systems. The final MAGIC data-set consists of $\sim 86$ hours of high quality data.

The MAGIC data processed with the MAGIC Analysis and Reconstruction Software (MARS) with the standard MARS analysis chain[4]. Additionally, we use SkyPrism, a three dimensional ( $2 \times$ spatial and energy) likelihood software for MAGIC data, specifically developed to perform morphological and spectral studies of extended sources[5]. SkyPrism allows for the independent modeling of multiple sources ideal of complex sources, hence it is ideal for regions such as the HESS J1912+101. To measure the extension of HESS J1912+101, the MAGIC data is binned into $125 \times 125$ equally spaced spatial bins covering the $2.5^{\circ} \times 2.5^{\circ}$ field of view and 7 equally logarithmically spaced energy bins from $200 \mathrm{GeV}$ to $30.9 \mathrm{TeV}$. HESS J1912+101 is then modeled independently and sequentially modeled with $2 \mathrm{D}$ radially symmetric Gaussian, a uniform ring, and projected shell defined by

$$
M(R)=A \times \begin{cases}\sqrt{R_{2}^{2}-R^{2}}-\sqrt{R_{1}^{2}-R^{2}} & R<R_{1} \\ \sqrt{R_{2}^{2}-R^{2}} & R_{1}<R<R_{2} \\ 0 & R>R_{2}\end{cases}
$$

where is $R_{1}, R_{2}$, and the center the central position of the extension are varied until the maximum log-likelihood is found.

Table 2: The grid scan results for HESS J1912+101 with MAGIC data. For the ring model and the projected shell model, $R_{1}$ and $R_{2}$ correspond to inner radius and outer radius. For the Gaussian model, $R_{2}$ corresponds to $\sigma_{68}$. The errors are $1 \sigma$ confidence level. $\log \mathscr{L}$ is the maximum log-likelihood.

\begin{tabular}{lccccc}
\hline \hline Model & $\mathrm{RA}\left[^{\circ}\right]$ & $\mathrm{DEC}\left[^{\circ}\right]$ & $R_{1}\left[^{\circ}\right]$ & $R_{2}\left[^{\circ}\right]$ & $\log \mathscr{L}$ \\
\hline Gaussian & $288.27_{-0.03}^{+0.04}$ & $10.17_{-0.03}^{+0.04}$ & - & $0.47_{-0.05}^{+0.03}$ & -110596 \\
Ring & $288.27_{-0.02}^{+0.03}$ & $10.17_{-0.04}^{+0.01}$ & $0.10_{-0.04}^{+0.03}$ & $0.46_{-0.01}^{+0.03}$ & -110589 \\
Projected shell & $288.27_{-0.03}^{+0.03}$ & $10.17_{-0.03}^{+0.02}$ & $0.26_{-0.06}^{+0.04}$ & $0.50_{-0.03}^{+0.03}$ & -110590 \\
\hline
\end{tabular}

The results of the maximum log-likelihood scan are shown in Table 2 where for the Radial Gaussian model $R_{2}$ corresponds to the $68 \%$ containment radius. The ring model is preferred over the Radial Gaussian model but it is statically indistinguishable from the projected shell model. The results of the extension fit is displayed on Figure 1 left. 
To estimate the flux of HESS J1912+101 from the MAGIC, data points are unfolded using a Tikhonov regularization term[15], an integration radius of $0.5^{\circ}$, and an energy threshold of $200 \mathrm{GeV}$. The standard MAGIC uncertainty in the energy scaling of $15 \%$ and $11-18 \%$ for the flux normalization are applied[13, 12]. The unfolded MAGIC data points in Figure 2 with black points.

\section{Fermi-LAT Analysis and Results}

Fermi-LAT is the primary instrument on board the Fermi Gamma Ray Space Telescope designed to detect $\gamma$-rays from $30 \mathrm{MeV}$ to $>500 \mathrm{GeV}$. It measures the entire sky every 3 hours[6]. We use 128 months (2008 August 4th to 2019 February 7th) of P8R3_SOURCE_V2 ${ }^{1}$ class data with ScienceTools version 11-07-00 ${ }^{2}$ and fermipy version 00-17-0 $0^{3}$ to perform the Fermi-LAT analysis. We analyze a $10^{\circ} \times 10^{\circ}$ region of interest (ROI) binned with $200 \times 200$ equally spaced spatial bins (a bin size of $\left.0.05^{\circ}\right)$ centered on $($ RA,DEC $)=\left(288.27^{\circ}\right.$ and $\left.10.17^{\circ}\right)$ and 26 equally logarithmically spaced energy bins from $1 \mathrm{GeV}$ to $2 \mathrm{TeV}$. Events are filtered with the standard data quality cut (DATA_QUAL $==1 \quad \& \& \quad L A T \_C O N F I G==1$ ) and events with zenith angle less than $105^{\circ}$ to remove Earth limb $\gamma$-ray contamination. Additionally, to mitigate effects from the recently discovered pulsations from PSR J1913+1011[7] we perform a phased analysis with an ephemerides provided by Jodrell Bank Observatory and remove on-peak events and scale the exposure accordingly.

Table 3: Results of the extension for HESS J1912+101 with Fermi-LAT data. RA and DEC are shown with statistical uncertainties. $R_{\text {ext }}$ corresponds to the $68 \%$ containment radius for Radial Gaussian and disk radius for Radial Disk. The first and second errors for position and $R_{\text {ext }}$ are the statistical and systematic uncertainties, respectively.

\begin{tabular}{lccccc}
\hline \hline Spatial Model & $\mathrm{RA}\left[^{\circ}\right]$ & $\mathrm{DEC}\left[^{\circ}\right]$ & $\mathrm{R}_{\mathrm{ext}}\left[{ }^{\circ}\right]$ & $\mathrm{TS}$ & $\mathrm{TS}_{\mathrm{ext}}$ \\
\hline Point Source & $288.34 \pm 0.03$ & $10.03 \pm 0.02$ & - & 192.66 & - \\
Radial Gaussian & $288.37 \pm 0.04$ & $10.03 \pm 0.04$ & $0.55 \pm 0.06 \pm 0.01$ & 294.32 & 101.66 \\
Radial Disk & $288.39 \pm 0.03$ & $9.99 \pm 0.03$ & $0.55 \pm 0.03 \pm 0.01$ & 284.56 & 91.89 \\
Spatial Map & 288.28 & 10.18 & - & 252.51 & 59.84 \\
\hline
\end{tabular}

Using the binned maximum log-likelihood, a model of the ROI is produced using the FermiLAT 8 Year Source List ${ }^{4}$ (FL8Y) and the standard Galactic (gll_iem_v06.fits) and isotropic (iso_P8R3_SOURCE_PSF0123_V2) diffuse model. Additionally, the Fermi-LAT data is further separated into the four PSF event types: PSF0, PSF1, PSF2, and PSF3, where PSF0 is the lowest quality and PSF3 is the highest quality, thereby improving the ability to detect and resolve extended sources. Three sources are found within the $3 \sigma$ contours of HESS J1912+101: FL8Y

\footnotetext{
${ }^{1}$ https://fermi.gsfc.nasa.gov/ssc/data/analysis/documentation/Cicerone/Cicerone_Data/LAT_DP.html

${ }^{2} \mathrm{https} / / /$ fermi.gsfc.nasa.gov/ssc/data/analysis/software/

${ }^{3}$ https://fermipy.readthedocs.io/en/latest/

${ }^{4}$ https://fermi.gsfc.nasa.gov/ssc/data/access/lat/fl8y/
} 
J1913.3+1018, FL8Y J1912.7+0959, and FL8Y J1914.6+1009, which are removed and replaced with a single point source located at the MAGIC position of HESS J1912+101 taken from Table 2. We then scan the extension of the single point source with a radially symmetric Gaussian, disk, and spatial map template where the spatial template is determine from the MAGIC contours shown in the right on Figure 1. Finally we determine the most statistically significant extension using:

$$
\mathrm{TS}_{\text {ext }}=2 \log \left(\mathscr{L}_{\text {ext }} / \mathscr{L}_{\mathrm{ps}}\right)
$$

where $\mathscr{L}_{\text {ext }}$ is the maximum log-likelihood of the respective model and $\mathscr{L}_{\mathrm{ps}}$ is the maximum loglikelihood of the point source[8].

The results of the extension test are seen in Table 3. While all morphologies are significantly detected, the best fit morphology is a radially symmetric Gaussian with an $R_{68}=0.55^{\circ} \pm 0.06^{\circ} \pm$ $0.01^{\circ}$ with statistical any systematic uncertainties respectively. To compare the Gaussian model to the point sources found in FL8Y, we use the Akaike Information Criteria (AIC) defined as:

$$
\mathrm{AIC}=2 k-2 \log \mathscr{L}
$$

where $k$ is the number of parameters and $\mathscr{L}$ is the likelihood of the model [9]. The difference in AIC between the Gaussian and the point source is 39.36 which shows the Gaussian model is preferred over the FL8Y points sources. The results of the extension can be seen in the right of Figure 1.

Using the Radial Gaussian spatial model, we calculate the flux and SED of HESS J1912+101 assuming a power-law as our model. For the spectral analysis, we use different settings to extend the spectrum to $100 \mathrm{MeV}$. We analyze a $14^{\circ} \times 14^{\circ}$ region of interest (ROI) binned with $140 \times 140$ equally spaced spatial bins centered on centered on the MAGIC position and 34 equally logarithmically spaced energy bins from $100 \mathrm{MeV}$ to $2 \mathrm{TeV}$. Above $1 \mathrm{GeV}$, the same event selection is applied as in the extension analysis. We omit all PSF0 data from $100 \mathrm{MeV}$ to $1 \mathrm{GeV}$ due to its poor PSF causing possible contamination from nearby sources. For PSF1, PSF2, and PSF3 data we only include photons with a zenith angle below $85^{\circ}, 95^{\circ}$, and $100^{\circ}$, respectively, from $100 \mathrm{MeV}$ to $1 \mathrm{GeV}$ to further reduce possible $\gamma$-ray contamination from the Earth limb due to the increase of Fermi-LAT PSF at lower energies.

To estimate spectral systematic uncertainty associated with incomplete understanding of the strong Galactic diffuse $\gamma$-ray emission in the HESS J1912+101 region, we use eight alternative Galactic and isotropic diffuse models (aIEMs). The aIEMs and method for calculating the systematic uncertainties are taken from the 1st Fermi-LAT Supernova Remnant Catalog[14]. To combine the results from the standard Galactic diffuse and the eight aIEMs, we use

$$
\sigma_{\mathrm{sys}, w}=\sqrt{\frac{1}{\sum_{i}^{M} w_{i}} \sum_{i}^{M} w_{i}\left(P_{i}-P_{\mathrm{STD}}\right)^{2}}
$$

where weights are $w_{i}=1 / \sigma_{i}^{2}$. The Fermi-LAT SED for HESS J1912+101 is shown with the red points in Figure 2.

\section{Discussion}

Figure 1 shows the morphology of HESS J1912+101 as seen by MAGIC (left) and Fermi-LAT (right). The preferred MAGIC morphology is that of a projected shell with $R_{1}=\left(0.26_{-0.06}^{+0.04}\right)^{\circ}$ and 

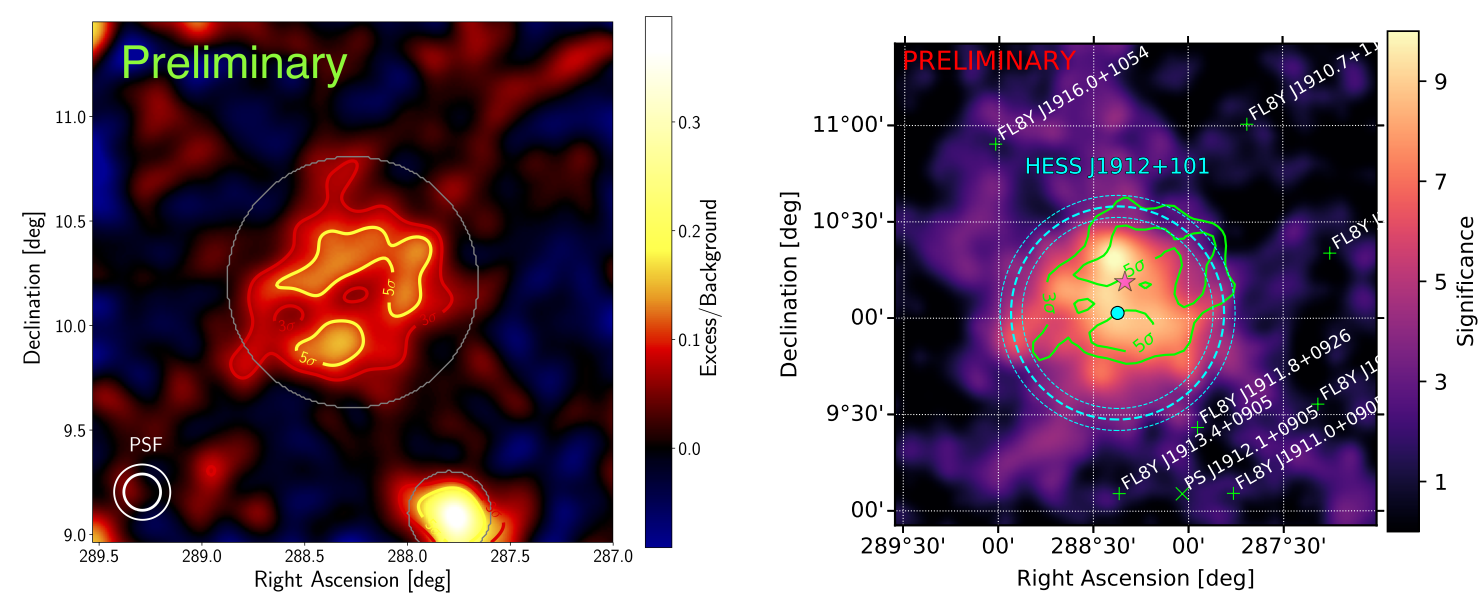

Figure 1: Left: The $2.5^{\circ} \times 2.5^{\circ}$ skymap of HESS J1912+101 showing the relative signal to background count rate from MAGIC data. The minimum energy for this skymap is $200 \mathrm{GeV}$. The gray circles are exclusion regions which include HESS J1912+101 and W 49B. The PSF shows the $39 \%$ and $68 \%$ containment radius. Right: The $2.5^{\circ} \times 2.5^{\circ}$ skymap of HESS J1912+101 showing the significance TS map from FermiLAT data. FL8Y sources are shown with green crosses. The center position and extension including total uncertainties of Radial Gaussian are shown with the filled cyan circle and dashed cyan circles respectively. Additionally, the MAGIC $3 \sigma$ and $5 \sigma$ are shown with the green contours.

$R_{2}=\left(0.50_{-0.03}^{+0.03}\right)^{\circ}$ centered at $($ RA,DEC $\left.)=\left(\left(288.27_{-0.03}^{+0.03}\right)^{\circ},\left(10.17_{-0.03}^{+0.02}\right)^{\circ}\right)\right)$. It should be noted that the projected shell model has the same significance as that of a uniform ring model for MAGIC data but we use the projected shell model since it provides a more physically motivated morphology than a uniform ring. The preferred Fermi-LAT morphology is that of a radially symmetric Gaussian with $R_{68}=(0.55 \pm 0.06 \pm 0.01)^{\circ}$, with statistical and systematic uncertainties, located at $($ RA,DEC $)=$ $\left((288.37 \pm 0.04)^{\circ},(10.03 \pm 0.04)^{\circ}\right)$ with statistical uncertainties. The locations of the center of the MAGIC and Fermi-LAT results are consistent within $2 \sigma$, being the Fermi-LAT position slightly displaced towards the southwest of the MAGIC position.

The MAGIC and H.E.S.S. morphologies are consistent within $1 \sigma$ when using the projectedshell model. The best spatial model for the Fermi-LAT data is the Gaussian model. This does point to a potential morphology change of HESS J1912+101 between the GeV and TeV energy ranges. This could be due to the gating of PSR J1913+1011 not entirely removing associated $\gamma$-ray emission.

The joint Fermi-LAT and MAGIC SED is shown in Figure 2. This represents a clear continuous spectrum from $100 \mathrm{MeV}$ to $30 \mathrm{TeV}$. We perform a joint forward folding fit with the Fermi-LAT, MAGIC and H.E.S.S data using a power-law (PL)

$$
\mathrm{PL}: \frac{\mathrm{d} F}{\mathrm{~d} E}=f_{0}\left(\frac{E}{E_{0}}\right)^{-\Gamma}
$$

and power-law with exponential cutoff (ECPL)

$$
\text { ECPL : } \frac{\mathrm{d} F}{\mathrm{~d} E}=f_{0}\left(\frac{E}{E_{0}}\right)^{-\Gamma} \exp \left(-\frac{E}{E_{\mathrm{cut}}}\right)
$$




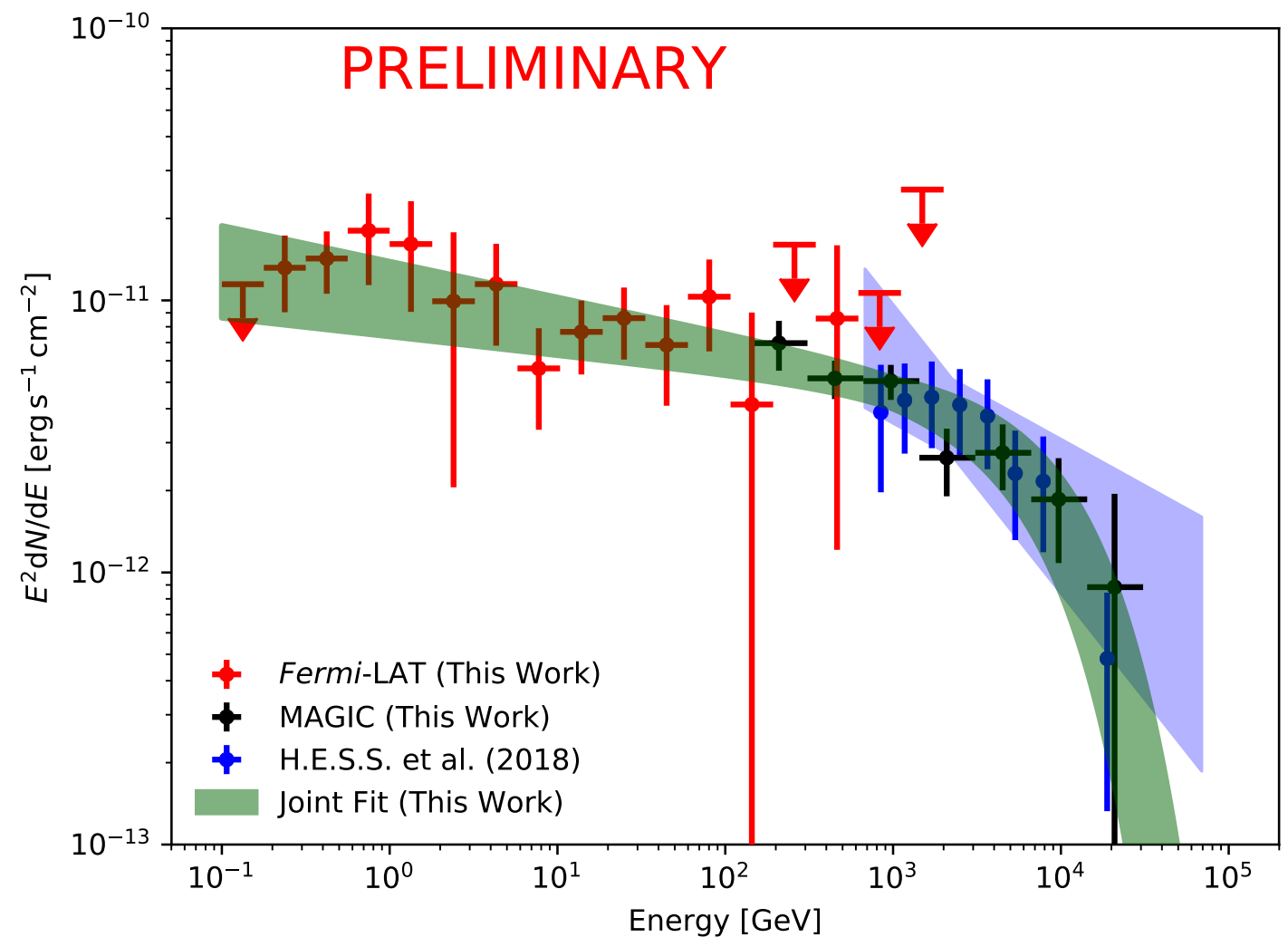

Figure 2: The MeV-TeV SED of HESS J1912+101. The red points: Fermi-LAT SED. The black points: unfolded MAGIC SED. The blue points: unfolded H.E.S.S. SED. The blue butterfly: the best fit PL function for H.E.S.S SED. The green line with the shaded region: the best fit ECPL function obtained by the joint forward folding fit.

where $f_{0}$ is the normalization, $E_{0}$ is the scale energy, $\Gamma$ is the spectral index, and $E_{\text {cut }}$ is the cutoff energy. For now, the joint fit is only considering correlations between the MAGIC data points. When fitting with a PL, we find an $f_{0}=\left(2.06_{-0.14}^{+0.15}\right) \times 10^{-12} \mathrm{~cm}^{-2} \mathrm{~s}^{-1} \mathrm{TeV}^{-1}$, an $\Gamma=2.20 \pm 0.02$ at $E_{0}=1 \mathrm{TeV}$. The PL fit has a $\chi^{2} / \mathrm{NDF}$ ( $p$-value $)=82.47 / 30\left(8.65 \times 10^{-7}\right)$, therefore we can exclude the PL with $4.9 \sigma$ significance. When fitting with an ECPL, we find an $f_{0}=\left(3.17_{-0.31}^{+0.33}\right) \times 10^{-12}$ $\mathrm{cm}^{-2} \mathrm{~s}^{-1} \mathrm{TeV}^{-1}$, an $\Gamma=2.09_{-0.02}^{+0.04}$ at $E_{0}=1 \mathrm{TeV}$, and $E_{\text {cut }}=9.59_{-2.47}^{+3.48} \mathrm{TeV}$. The ECPL fit has a $\chi^{2} / \mathrm{NDF}(p$-value $)=34.09 / 29(0.24)$ and much better represents the data. This joint ECPL fit is shown with the green shaded area in Figure 2.

From the morphological and spectral information across 7 decades in energy, it is most likely that HESS J1912+101 is a supernova remnant (SNR) as opposed to a pulsar wind nebula. The flat spectral index of $2.09_{-0.02}^{+0.04}$, shell-like morphology in TeV energies, cutoff energy of $9.59_{-2.47}^{+3.48} \mathrm{TeV}$ are all consistent with a SNR of an age of $\sim 10^{3}$ years in the Sedov-Taylor phase of evolution. Assuming a distance of $1.5 \mathrm{kpc}$ to HESS J1912+101 would give a radius of $\sim 13 \mathrm{pc}$, also well within agreement of other SNRs. Deeper particle population modeling and further multi-wavelength observations in radio and X-ray would further confirm the nature of HESS J1912+101. 


\section{References}

[1] Aharonian, F., Akhperjanian, A. G., Barres de Almeida, U., et al. 2008, Astronomy and Astrophysics, 484, 435

[2] H. E. S. S. Collaboration, Abdalla, H., Abramowski, A., et al. 2018, Astronomy and Astrophysics, 612, A8

[3] V. P. Fomin, A. A. Stepanian, R. C. Lamb, D. A. Lewis, M. Punch and T. C. Weekes, Astropart. Phys. 2, 137 (1994). doi:10.1016/0927-6505(94)90036-1

[4] Moralejo, A., Gaug, M., Carmona, E., et al. 2009, arXiv e-prints, arXiv:0907.0943

[5] Vovk, I., Strzys, M., \& Fruck, C. 2018, Astronomy and Astrophysics, 619, A7

[6] Atwood, W. B., Abdo, A. A., Ackermann, M., et al. 2009, The Astrophysical Journal, 697, 1071

[7] Smith, D. A., Bruel, P., Cognard, I., et al. 2019, The Astrophysical Journal, 871, 78

[8] Lande, J., Ackermann, M., Allafort, A., et al. 2012, The Astrophysical Journal, 756, 5

[9] Akaike, H. 1974, IEEE Transactions on Automatic Control, 19, 716

[10] Atwood, W., Albert, A., Baldini, L., et al. 2013, arXiv e-prints, arXiv:1303.3514

[11] Bruel, P., Burnett, T. H., Digel, S. W., et al. 2018, arXiv e-prints, arXiv:1810.11394

[12] Aleksić, J., Ansoldi, S., Antonelli, L. A., et al. 2016, Astroparticle Physics, 72, 76

[13] Aleksić, J., Alvarez, E. A., Antonelli, L. A., et al. 2012, Astroparticle Physics, 35, 435

[14] Brandt, T. J., Acero, F., de Palma, F., et al. 2015, arXiv e-prints, arXiv:1507.03633

[15] Ramm, A. G., \& Meister, E. 1981, Mathematical Methods in the Applied Sciences, 3, 336 